\title{
“Shooting a Bird": Game System Using Facial Feature for the Handicapped People
}

\author{
Jinsun Ju, Yunhee Shin, and Eun Yi Kim \\ Department of Internet and Multimedia Engineering, Konkuk Univ., Korea \\ \{vocaljs, ninharsa, eykim\}@konkuk.ac.kr
}

\begin{abstract}
This paper presents a novel computer game system that controls a game using only the movement of human's facial features. Our system is specially designated for the handicapped people with severe disabilities and the people without experience of using the computer. Using a usual PC camera, the proposed game system detects the user's eye movement and mouse movement, and then interprets the communication intent to play a game. The game system is tested with 42 numbers of people, and then the result shows that our game system should be efficiently and effectively used as the interface for the disabled people.
\end{abstract}

Keywords: Augmented game, HCI, Facial feature tracking, neural network.

\section{Introduction}

Recently, computer games using traditional interface such as a keyboard and a mouse have been replaced by new game paradigm such as body-interaction games. The body-interaction games use human's gestures to control a game, which makes the players feel more realistic enjoyment and actual feelings. From now on, various interfaces based on human gestures have been developed to provide the natural communication in between players and game systems. Some systems use the hand gestures as the input to control a game and some systems use the full-body motions.

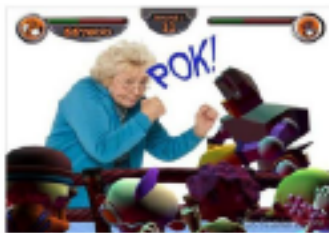

(a)

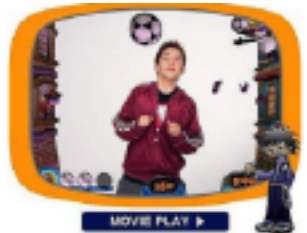

(b)

Fig. 1. (a) and (b) Eyetoy ${ }^{\mathrm{TM}}$ game system

Although such systems are well worked on the people without physical disabilities, they can not be applied to the handicapped people. For the handicapped people, the game system with general interface that adaptable to the people with various physical disabilities should be developed. 
Accordingly, this paper presents a novel computer game system that controls a game using only the movement of human's facial features. Our system is specially designated for the handicapped people with severe disabilities and the people without experience of using the computer. Using a usual PC camera, the proposed game system detects the user's eye movement and mouse movement, and then interprets the communication intent to play a game.

The game system is tested with 42 numbers of people, and then the result shows that our game system should be efficiently and effectively used as the interface for the disabled people.

The organization of the paper is as follows. Section 2 gives an overview of our game system. Section 3 describes our game module ("Shooting a bird") Section 4 describes our interface (feature detector, feature tracker, mouse controller). Experimental results are presented in Section 5 and the final conclusions are made in Section 6.

\section{Overview the Game System}

The outline of the proposed game system is demonstrated in Fig. 2. Fig. 2(a) shows our game system, which consists of a PC camera and a computer. The PC camera, which is connected to the computer through the USB port, supplies 30 color images of size $320 \times 240$ per second. The computer is a PentiumIV-3GHz with the Window XP operating system.

Fig.4(b) overviews of our game system. The game is made using FLASH(version MX 2004), and is one of the shooting games where a user hits randomly flying bird with a gun. A player controls the cursor by his (or her) eye movement and fires a gun by opening his mouth. Our game movements of a player are detected and tracked in the interface modular, which will be described in Section 4 in detail.

Our game movements of a player are detected and tracked in the interface modular, which will be described in Section 4 in detail.

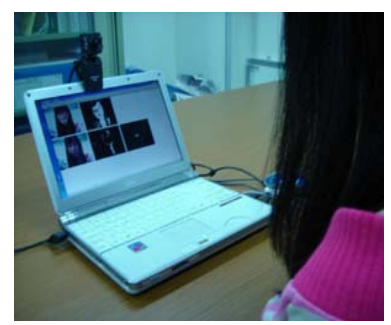

(a)

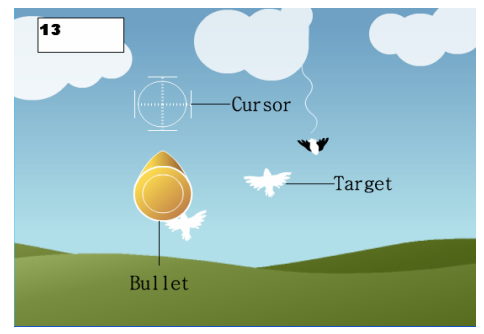

(b)

Fig. 2. "Shooting a bird game" (a) hardware architecture (b) Shot of "shooting a bird"

\section{Game Module}

Fig. 3 shows a shot of our game system. The game is made using FLASH(version MX 2004).The game is one of the shooting games where a user hits randomly flying bord 


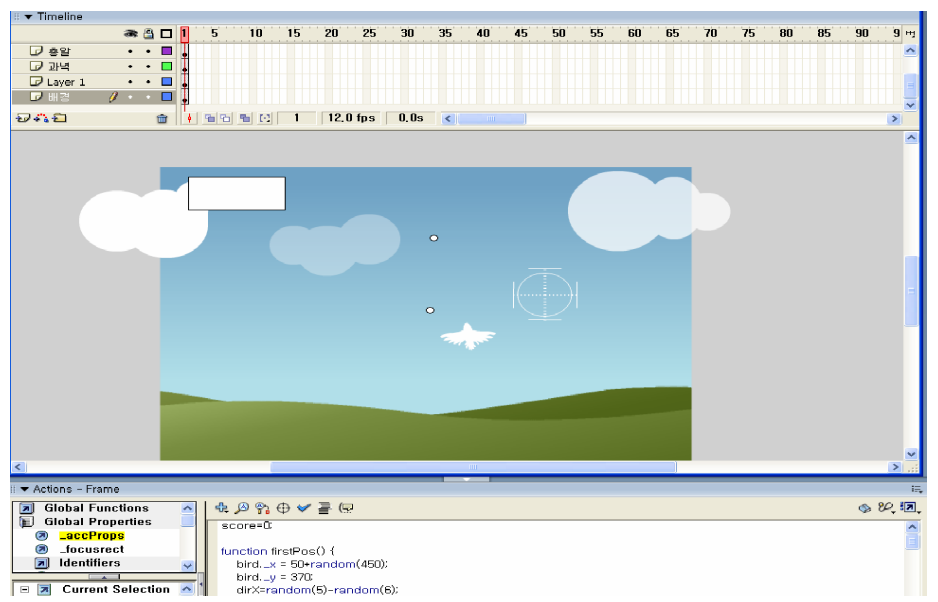

Fig. 3. shot of "shooting a bird"

with a gun. The game consists of three parts. 1) Characters movement. 2) a movement of background. 3) Calculated score. A player controls the cursor by his (of her) eye movement and fires a gun by opening his mouth. These movements of a player are detected and tracked in the interface modular.

\section{Interface Module}

Fig.4 demonstrates the interface module, which consists of facial feature detector, facial feature tracker and mouse controller. The facial feature detector firstly extracts user's face from the background using skin-color model, and then localizes user's eyes and mouth from the face region. To be robust to the complex background and users with various physical conditions, eye regions are localized using neural network (NN)-based texture classifier that discriminates the facial region into eye class and non-eye class, and then the mouth region is detected based on edge information. Once these features are extracted, they are continuously tracked by facial feature tracker: a mean-shift algorithm is used for eye tracking, and a template matching is used for the

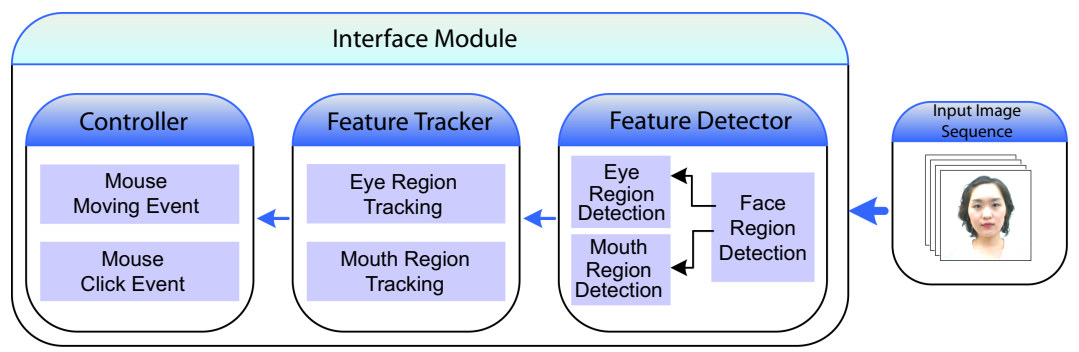

Fig. 4. The interface to use eye and mouse movement 
mouth tracking. Based on the tracking results, mouse operations such as movement or click are implemented in a mouse controller. Our system moves the cursor to the point at which the user gazed on the display, and then fires the gun to that point if he opens and closes his mouth.

\subsection{Facial Feature Detector}

In facial feature detector, given an image sequence, the regions of the face and facial features are detected automatically. The facial region is first obtained using skin-color and then, the eye regions are localized by a neural network (NN)-based texture classifier that discriminates each pixel in the extracted facial regions into the eye-class and non-class using the texture property. And then mouth is localized by edges information within the search region estimated using several heuristic rules based on eye position. The detection results are delivered to feature tracker.

\subsection{Facial Features Tracker}

In facial feature tracker, it tracks eyes and mouth. Once the eye and mouth regions are localized in feature detector, they are continuously and correctly tracking by meanshift algorithm and template matching, respectively.

Fig. 5 shows the results of the facial features tracking, where the facial features are drawn out white for the better viewing. As can be seen in Fig. 5 the facial features are accurately tracking. The extracted facial features have been drawn white for better viewing. The features are tracked throughout the 100 frames and not lost once.

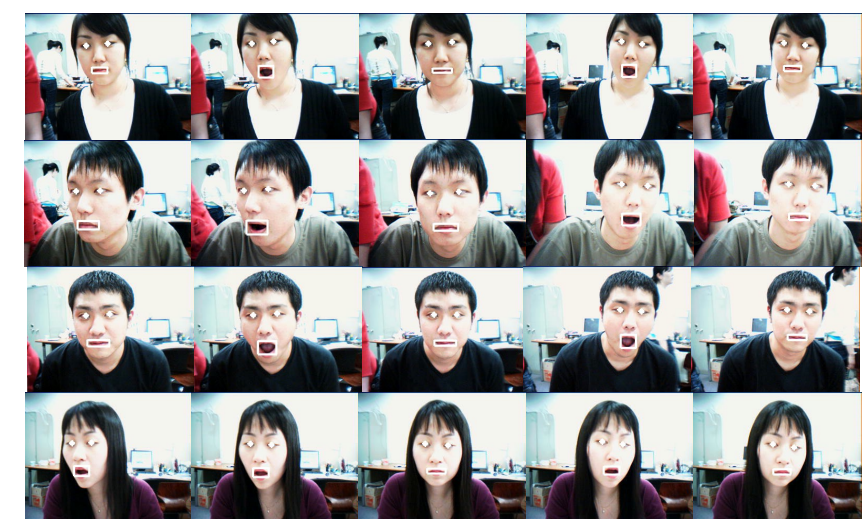

Fig. 5. Tracking result of facial features

\subsection{Mouse Control Module}

The computer translates the user's eye movements into the mouse movements by processing the images received from the PC camera. Then the processing of a video sequence is performed by the proposed facial features tracking system. The system determines the center of eyes in the first frame as the initial coordinates of mouse, and 
then computes it automatically in subsequent frames. The coordinates of mouth region and eye regions in each frame are sent to the operating system through Window functions. Mouse point is moved according to eye movement.

\section{Experimental Results}

We use these facial features tracking method as an interface to control the game system. Twenty handicapped peoples tested our system. Fig. 6 shows the snapshot to play a game. A player was playing our game using his (or her) facial features. Fig.8 is a player controls the cursor by his (or her) eye movement and fires a gun by opening his mouth. These movements of a player are detected and tracked in the interface modular. Based on the results, it does the mouse operations such as movement or click.

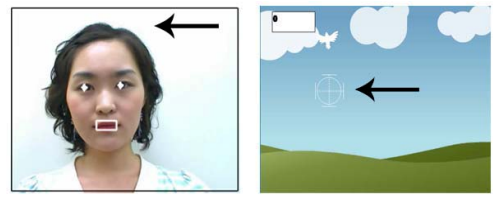

(a)

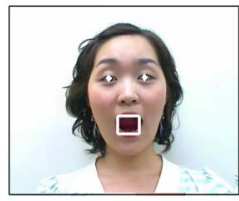

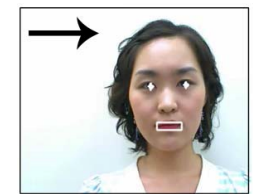

(b)

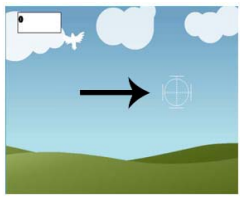

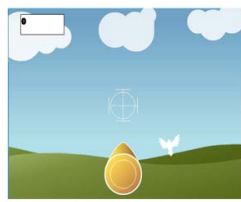

(c)

Fig. 6. The interface using our game system

Table 1. "Shooting a bird" Game test result (s)

\begin{tabular}{ccccc}
\hline & \multicolumn{2}{c}{ The people } & \multicolumn{2}{c}{$\begin{array}{c}\text { The People } \\
\text { Disabled users }\end{array}$} \\
& Mean & Deviation & Mean & Deviation \\
Shooting a bird game & 95.25 & 14.02 & 188.94 & 76.56 \\
Standard Mouse & 48.6 & 3.14 & - & - \\
\hline
\end{tabular}

Table 1 presents the averages times to be taken to shooting 10-birds in the "shooting a bird" game. To show the effectiveness of the game system, it was applied to the disabled users. As the disabled users cannot move their hands, they tested with only our interface system. There are two interesting points in this table. In case of nondisabled users, the average time taken to play the game with the standard mouse was the faster than the proposed interface. Then, the first noticeable point is that the deviation of our interface is larger than one of standard mouse. This difference is resulted 
in the fact that they are accustomed to standard mouse but they are poor to using our interface. Consequently, that result tells us that the computational time can be reduced if they would practice using our game system. The second interesting point is that the deviation in the disabled users is larger than one of the non-disabled users when playing the game with our interface system. This difference was the same reason as with the first case. Most of the non-disabled users are accustomed to playing a computer game, while a small number of disabled users have experienced the computer game. That is, the time to play a game could be rapidly reduced if the disabled users would have the sufficient practice.

Moreover, to assess the validity of the proposed system for disabled users, it was applied to the "spelling board."

Table 2. Spelling board result $(s)$

\begin{tabular}{ccccc}
\hline & \multicolumn{2}{c}{ Group 1 } & \multicolumn{2}{c}{ Group 2 } \\
\cline { 2 - 5 } & Mean & Deviation & Mean & $\begin{array}{c}\text { Deviatio } \\
\mathrm{n}\end{array}$ \\
\hline \hline Eye Mouse[] & 30.6 & 4.76 & 63.94 & 32.11 \\
\hline Proposed method & 16.95 & 4.19 & 37.12 & 18.40 \\
\hline Standard Mouse & 8.4 & 0.44 & $\times$ & $\times$ \\
\hline
\end{tabular}

In this experiment, the clicking event was mainly involved, like the "Shooting a bird" game system. The timing results for the experiments are summarized in table 2 .

The two experiment results show that our systems are effectively used for the disabled users.

Consequently, the experiment with proposed interface showed that it has a potential to be used as generalized user interface in many applications.

\section{Conclusions}

In this paper, we implemented the game system for the handicapped people using multiple facial features detection and tracking as PC-based HCI system. The proposed game system has worked very well in a test database and cluttered environments with 42-people. Then it is accurately detected and tracked to facial features regardless of disabilities or not and also is robust to the time-varying illumination and less sensitive to the specula reflection of eyeglasses.

In experiment is compared the proposed method with standard mouse respectively. The experimental results show that our system should be efficiently and effectively used as the interface for the disabled people. Also our system has a potential to be used as generalized user interface in many application.

\section{Acknowledgments}

This work was supported by Seoul R\&BD Program in Korea. 


\section{References}

1. Sharma, R., Pavlovic, V.I., Huang, T.S.: Toward multimodal human-computer interface. Proceedings of the IEEE 86, 853-869 (1998)

2. Scassellati, B.: Eye finding via face detection for a foveated, active vision system. American Association for Artificial Intelligence (1998)

3. Hornof, A., Cavender, A., Hoselton, R.: EyeDraw: A System for Drawing Pictures with Eye Movements. ACM SIGACCESS Accessibility and Computing, Issue 77-78 (2003)

4. Kim, E.Y., Kang, S.K. (eds.): ICCSA 2006. LNCS, vol. 3982, pp. 1200-1209. Springer, Heidelberg (2006)

5. Lyons, M.L.J.: Facial Gesture Interfaces for Expression and Communication. In: IEEE International Conference on Systems, Man, Cybernetics, vol. 1, pp. 598-603 (2004)

6. Jie, Y., DaQuan, Y., WeiNa, W., XiaoXia, X., Hui, W.: Real-time detecting system of the driver's fatigue. In: ICCE International Conference on Consumer Electronics, 2006 Digest of Technical Papers. pp. 233-234 (2006)

7. Schiele, B., Waibel, A.: Gaze Tracking Based on Face-Color. School of Computer Science. Carnegie Mello University (1995)

8. Chan, a.d.c., Englehart, K., Hudgins, B., Lovely, D.F.: Hidden markov model classification of myoeletric signals in speech. IEEE Engineering in Medicine and Biology Magazine 21(4), 143-146 (2002)

9. Comaniciu, D., Ramesh, V., Meer, P.: Kernel-Based Object Tracking. IEEE Trans. Pattern Analysis and Machine Intelligence 25(5), 564-577 (2003)

10. Olson, C.F.: Maximum-Likelihood Template Matching. In: Proc. IEEE Conf. Computer Vision and Pattern Recognition, vol. 2, pp. 52-57 (2000)

11. Shin, Y., Kim, E.Y.: Welfare Interface Using Multiple Facial Feature Tracking. In: Sattar, A., Kang, B.-H. (eds.) AI 2006. LNCS (LNAI), vol. 4304, pp. 453-462. Springer, Heidelberg (2006) 